\title{
Implications of Discretization on Dissipativity and Economic Model Predictive Control
}

\author{
Olumuyiwa I. Olanrewaju*, Jan M. Maciejowski \\ Department of Engineering, University of Cambridge, Trumpington St, Cambridge \\ CB2-1PZ, UK
}

\begin{abstract}
Economic model predictive control, where a generic cost is employed as the objective function to be minimized, has recently gained much attention in model predictive control literature. Stability proof of the resulting closed-loop system is often based on strict dissipativity of the system with respect to the objective function. In this paper, starting with a continuous-time setup, we consider the 'discretize then optimize' approach to solving continuous-time optimal control problems and investigate the effect of the discretization process on the closedloop system. We show that while the continuous-time system may be strictly dissipative with respect to the objective function, it is possible that the resulting closed-loop system is unstable if the discrete-approximation of the continuoustime optimal control problem is not properly set up. We use a popular example from the economic MPC literature to illustrate our results.

Keywords: Economic Model Predictive Control, Dissipativity, Stability, Discretization, Direct Collocation
\end{abstract}

\section{Introduction}

Economic model predictive control (e-MPC), a model predictive control (MPC) approach to the optimal control of systems has recently gained much

\footnotetext{
${ }^{*}$ Corresponding author. Tel.: +44 1223748357

Email addresses: oio24@cam.ac.uk (Olumuyiwa I. Olanrewaju), jmm@eng.cam.ac.uk (Jan M. Maciejowski)
} 
popularity. The main difference between e-MPC and existing MPC approaches is the nature of the objective function being optimized. While conventional MPC approaches employ a positive-definite function that minimizes deviations from desired set-point, the objective function in e-MPC is a generic cost that is related to the economics of the system's operation.

Two major techniques exist in the literature for designing digital controllers for systems: the emulation method and the direct design method [1-3]. In the emulation method, the controller design is done in the continuous-time domain followed by a discretization of the controller. In the direct design methodology, the digital controller is designed directly using a discretized model or a discrete approximation of the system. While emulation methods do exist for MPC, most standard MPC settings use a direct design that involves discretizing both the system's model and objective function.

Of great importance in the context of economic-MPC is the dissipativity of the system with respect to the economic objective as this is one of the conditions on which the stability proof for the closed-loop system is often based. It has been shown $[4,5]$ that strict dissipativity of the system with respect to the economic cost plays a central role in the stability proofs of the closed loop system. It has also been shown in the economic-MPC literature that if a system is dissipative with respect to the economic objective, then static equilibrium operation of the system is optimal [5-7]. It is therefore important to know what happens to the dissipativity property when the continuous-time setup is 'discretized then optimized' as done in the direct design method. Is it possible to have a dissipative continuous-time setup and the discretized setup not dissipative? Under what circumstances can these happen and how can we avoid them?

These are the questions we raise and attempt to answer in this paper. We show that due to the approximate discretization of the underlying continuoustime optimal control problem, it is possible to lose the system's dissipativity (with respect to the given economic objective) hence, possible loss of stability of the closed-loop system. We also show that the conventional MPC scheme (where the cost function is designed to be positive-definite) is immune to such 
behaviour.

This paper is structured as follows: Section 2 introduces the problem statement. The effect of approximate discretization on a linear-quadratic optimal control problem is discussed in Section 3. The effect of approximate discretization is extended to direct transcription methods for solving optimal control problems in Section 4 with the focus on direct collocation methods. Section 5 contains a popular example from e-MPC literature while Section 6 concludes the paper.

\section{Preliminaries}

In this paper, we consider the continuous-time, finite horizon optimal control problem

$$
\begin{aligned}
& J_{T}^{*}(x)=\min _{\mathbf{u}} J(x, \mathbf{u}) \triangleq \int_{0}^{T} l_{c}(x(t), u(t)) \mathrm{d} t \\
& \text { subject to }\left\{\begin{array}{l}
\dot{x}(t)=f(x(t), u(t)) \forall t \in[0, T] \\
x(t) \in \mathbb{X}, u(t) \in \mathbb{U}, \forall t \in[0, T] \\
x(0)=x_{0}, \quad x(T)=x_{s}
\end{array}\right.
\end{aligned}
$$

where $x_{0}$ is the initial condition and the pair $\left(x_{s}, u_{s}\right)$ that satisfies

$$
l_{c}\left(x_{s}, u_{s}\right)=\min _{x, u}\left\{l_{c}(x, u) \mid f(x, u)=0, x \in \mathbb{X}, u \in \mathbb{U}\right\}
$$

is defined as the optimal static equilibrium. The constraint sets $\mathbb{X} \subseteq \mathbb{R}^{n}, \mathbb{U} \subset$ $\mathbb{R}^{m}$ with $\mathbb{X} \times \mathbb{U}$ assumed compact. This optimal control problem is at the core of model predictive control of systems where (1) is carried out in a receding horizon manner. The cost function $l_{c}(x(t), u(t))$ here is assumed generic. The direct design approach to solving (1) is to discretize it by the use of exact or approximate discretization methods, and then optimize.

As stated in section 1, dissipativity of a system with respect to the given cost function is important in the context of e-MPC as the stability proof of the optimal controlled system often relies on this property. Hence, given a 'dissipative' continuous-time setup that is approximately discretized, it is imperative 
to know if dissipativity is preserved. A review of the literature on preservation of dissipativity shows that most of the work done is on finding supply rates for which the discretized system (controller) is passive while some viewed dissipativity preservation as preservation of positive-realness of the system [8-11]. However, dissipativity of the system as applied in e-MPC is with respect to a given running (stage) cost. Hence, given a continuous-time system that is dissipative with respect to a given supply rate, we seek to find out if this dissipativity is preserved in the discretized setup and in cases where the explicit discretized form is not available, how discretization affects the closed-loop system's stability.

For analysis purpose, we consider linear systems with quadratic running costs without restrictions on the definiteness of the cost. The origin is taken to be the optimal static equilibrium. The use of a linear-quadratic setup makes for ease of checking the dissipativity condition.

Definition 1. Consider the continuous-time system

$$
\dot{x}=A_{c} x+B_{c} u
$$

and the running cost

$$
l_{c}(x, u)=x^{T} Q_{c} x+u^{T} R_{c} u+2 x^{T} S_{c} u
$$

System (3) is said to be dissipative [12, 13] with respect to running cost (4) if there exists a quadratic storage function, $V(x)=x^{T} P_{c} x$ where $P_{c}=P_{c}^{T}$ such that for all $x \in \mathbb{X}, u \in \mathbb{U}$,

$$
\dot{x}^{T} P_{c} x+x^{T} P_{c} \dot{x} \leq l_{c}(x, u) .
$$

This is implied by the existence of a $P_{c}=P_{c}^{T}$ such that the Linear Matrix Inequality (LMI)

$$
\left[\begin{array}{cc}
A_{c}^{T} P_{c}+P_{c} A_{c}-Q_{c} & P_{c} B_{c}-S_{c} \\
B_{c}^{T} P_{c}-S_{c}^{T} & -R_{c}
\end{array}\right] \leq 0
$$

is feasible. If (6) holds with strict inequality, the system is said to be strictlydissipative with respect to the running cost. 
Definition 2. The discrete time system

$$
x_{k+1}=A x_{k}+B u_{k}
$$

is said to be dissipative $[5,13,14]$ with respect to the stage cost

$$
l_{d}\left(x_{k}, u_{k}\right)=x_{k}^{T} Q x_{k}+u_{k}^{T} R u_{k}+2 x_{k}^{T} S u_{k}
$$

if there exists a quadratic storage function $x_{k}^{T} P_{d} x_{k}$ where $P_{d}=P_{d}^{T}$ such that for all $x_{k} \in \mathbb{X}, u_{k} \in \mathbb{U}$ and $k \geq 0$,

$$
x_{k+1}^{T} P_{d} x_{k+1}-x_{k}^{T} P_{d} x_{k} \leq l_{d}\left(x_{k}, u_{k}\right)
$$

This is implied by the existence of a $P_{d}=P_{d}^{T}$ such that the LMI

$$
\left[\begin{array}{cc}
A^{T} P_{d} A-P_{d}-Q & A^{T} P_{d} B-S \\
B^{T} P_{d} A-S^{T} & B^{T} P_{d} B-R
\end{array}\right] \leq 0
$$

is feasible. If (10) holds with strict inequality, the system is said to be strictlydissipative.

We note that compactness of the constraint set and continuity of the quadratic storage function imply lower boundedness of the storage function, which is required for dissipativity to hold. Hence, $P_{c}$ and $P_{d}$ can be non-negative, provided the storage function remains lower bounded $[4,5,15-17]$.

Assumption 2.1. The continuous-time system (3) is dissipative with respect to the running cost (4).

\section{Effect of sampling Period on First Order Approximation}

In this section, we analyse the effect of the approximate discretization of optimal control problem (1) when the dynamics is linear and the cost function is quadratic. We note that there are established methods of computing the exact discrete equivalent of (1) when dealing with a linear-quadratic setup [18, 19]. Hence while one may not necessarily use an approximate method in the linear-quadratic case, analysing the effect of approximate discretization in the 
linear quadratic case will help understand the observed behaviour in the generic case. We start with the exact discretization method under the assumption of piecewise-constant inputs and take its first order approximation. This gives an explicit form of the discrete setup in terms of the continuous-time setup hence analysis linking both can be easily made.

Consider the continuous-time system (3) and running cost (4). Define

$$
\Phi(t)=e^{A_{c} t}, \Gamma(t)=\int_{0}^{t} e^{A_{c} \eta} B_{c} \mathrm{~d} \eta .
$$

Given a sampling time $t_{s}>0$, let (7) and (8) in Definition 2 be obtained by the exact discretization of (3) and (4) (under the assumption of piecewise-constant inputs i.e., zero-order-hold $(\mathrm{ZOH}))$ such that:

$$
\begin{aligned}
& A=\Phi\left(t_{s}\right)=I_{n_{x}}+A_{c} t_{s}+\frac{1}{2 !} A_{c}^{2} t_{s}^{2}+\frac{1}{3 !} A_{c}^{3} t_{s}^{3}+\ldots \\
& B=\int_{0}^{t_{s}} e^{A_{c} t} B_{c} \mathrm{~d} t=B_{c} t_{s}+\frac{1}{2 !} A_{c} B_{c} t_{s}^{2}+\frac{1}{3 !} A_{c}^{2} B_{c} t_{s}^{3}+\ldots \\
& {\left[\begin{array}{cc}
Q & S \\
S^{T} & R
\end{array}\right]=\int_{0}^{t_{s}}\left[\begin{array}{cc}
\Phi^{T}(t) & 0 \\
\Gamma^{T}(t) & I_{n_{x}}
\end{array}\right]\left[\begin{array}{cc}
Q_{c} & S_{c} \\
S_{c}^{T} & R_{c}
\end{array}\right]\left[\begin{array}{cc}
\Phi(t) & \Gamma(t) \\
0 & I_{n_{x}}
\end{array}\right] \mathrm{d} t}
\end{aligned}
$$

where $n_{x}$ is the number of states. Expanding the discrete cost from (11) in powers of $t_{s}$ yields the series expansion;

$$
\begin{aligned}
Q & =Q_{c} t_{s}+\frac{1}{2}\left(Q_{c} A_{c}+A_{c}^{T} Q_{c}\right) t_{s}^{2}+\ldots \\
R & =R_{c} t_{s}+\frac{1}{2}\left(S_{c}^{T} B_{c}+B_{c}^{T} S_{c}\right) t_{s}^{2}+\ldots \\
S & =S_{c} t_{s}+\frac{1}{2}\left(A_{c}^{T} S_{c}+Q_{c} B_{c}\right) t_{s}^{2}+\ldots
\end{aligned}
$$

Taking the first order approximation of (11) and (12) gives

$$
A=I_{n_{x}}+A_{c} t_{s}, B=B_{c} t_{s}, Q=Q_{c} t_{s}, R=R_{c} t_{s}, S=S_{c} t_{s} .
$$

where it is assumed that $t_{s}$ is in the set of all sampling periods that preserve stabilizability and detectability of the system. Substituting for $\{A, B, Q, R, S\}$, the dissipativity inequality (10) can be written in terms of the continuous-time setup as

$$
\left[\begin{array}{cc}
\left(I+A_{c}^{T} t_{s}\right) P_{d}\left(I+A_{c} t_{s}\right)-P_{d}-Q_{c} t_{s} & \left(I+A_{c}^{T} t_{s}\right) P_{d} B_{c} t_{s}-S_{c} t_{s} \\
\left(\left(I+A_{c}^{T} t_{s}\right) P_{d} B_{c} t_{s}-S_{c} t_{s}\right)^{T} & B_{c}^{T} t_{s} P_{d} B_{c} t_{s}-R_{c} t_{s}
\end{array}\right] \leq 0
$$


Dividing through by $t_{s}$ and rearranging yields

$$
\left[\begin{array}{cc}
P_{d} A_{c}+A_{c}^{T} P_{d}-Q_{c} & P_{d} B_{c}-S_{c} \\
B_{c}^{T} P_{d}-S_{c}^{T} & -R_{c}
\end{array}\right] \leq-t_{s}\left[\begin{array}{ccc}
A_{c}^{T} P_{d} A_{c} & A_{c}^{T} P_{d} B_{c} \\
B_{c}^{T} P_{d} A_{c} & B_{c}^{T} P_{d} B_{c}
\end{array}\right]
$$

If there exists $P_{d}$ that makes (13) feasible for a given $t_{s}$, then dissipativity of the system using this approximation is preserved. Furthermore given $\left\{A_{c}, B_{c}, Q_{c}, R_{c}, S_{c}\right\}$, an interval bisection approach can be applied to find values of $t_{s}$ for which a feasible $P_{d}$ exists.

We now describe an algorithm for finding possible values of $t_{s}$ for which a $P_{d}$ exists that guarantees feasibility of (13).

\section{Algorithm 3.1.}

1. Since the sampling time cannot be negative, set the lower bound on $t_{s}$ as 0 and the upper bound as $\frac{t_{r}}{4}$ where $t_{r}$ is the rise time of the continuous-time system. This upper bound on $t_{s}$ is chosen to ensure that there are at least 4 samples per rise time of the open-loop system [2].

2. Apply interval bisection to find the maximum $t_{s}$ for which a symmetric $P_{d}$ exists such that (13) is feasible. This is $t_{u}$, the upper bound on the set of $t_{s}$ that preserve dissipativity of the first order approximation.

3. Set the upper bound on $t_{s}$ as $t_{u}$ and apply interval bisection to find the minimum $t_{s}$ for which a symmetric $P_{d}$ exists such that (13) is feasible. This is $t_{l}$, the lower bound on the set of $t_{s}$ that preserve dissipativity of this first order approximation.

Remark 1. It is possible that the set of sampling periods for which a symmetric $P_{d}$ that satisfies (13) is not connected. Hence it is good practice to search, using Algorithm 3.1 iteratively by setting random $t_{s} \in(0, T]$ as the upper bound in the algorithm, till possible dissipativity-preserving $t_{s}$ have been found.

Remark 2. This algorithm is for the first order approximation of (11) under zero-order-hold. It is possible that no $t_{s}$ exists that preserves dissipativity under this approximation. This does not however imply that the discretized setup cannot be dissipative. Indeed it is expected that when the system is under zeroorder-hold, for $t_{s} \in(0, T]$, the discretized setup should be dissipative since the 
discretized setup under $\mathrm{ZOH}$ is an exact representation of the continuous-time setup.

Next, we show that while it is possible that no $t_{s}$ exists that preserves dissipativity when using an indefinite running cost (under first order approximation), dissipativity is always preserved with the use of a positive (semi)definite running cost.

Lemma 3.2. Consider (3). If $\left[\begin{array}{ll}Q_{c} & S_{c} \\ S_{c}^{T} & R_{c}\end{array}\right] \geq 0$, then $P_{c}=0$ satisfies (6) i.e (3) is guaranteed to be dissipative with respect to (4).

Proof. By substituting $P_{c}=0$ into (6), we have

$$
-\left[\begin{array}{ll}
Q_{c} & S_{c} \\
S_{c}^{T} & R_{c}
\end{array}\right] \leq 0
$$

which holds since $\left[\begin{array}{ll}Q_{c} & S_{c} \\ S_{c}^{T} & R_{c}\end{array}\right] \geq 0$.

Theorem 3.3. Consider the continuous-time system (3) with running cost (4). Then its discrete-time version (7) and (8) is always dissipative irrespective of the sampling time $t_{s}$ when using the zero-order-hold discretization method or its first order approximation provided $\left[\begin{array}{cc}Q_{c} & S_{c} \\ S_{c}^{T} & R_{c}\end{array}\right] \geq 0$.

Proof.

\section{First order approximation}

The discretized setup is dissipative if there exists $P_{d}$ and $t_{s}$ pair for which (13) is feasible. Using Lemma 3.2, existence of $P_{d}$ that satisfies this condition can be confirmed by substituting $P_{d}=0$ into (13) which yields

$$
\left[\begin{array}{ll}
-Q_{c} & -S_{c} \\
-S_{c}^{T} & -R_{c}
\end{array}\right] \leq-t_{s}\left[\begin{array}{ll}
0 & 0 \\
0 & 0
\end{array}\right]=0
$$

This is satisfied since $\left[\begin{array}{ll}Q_{c} & S_{c} \\ S_{c}^{T} & R_{c}\end{array}\right] \geq 0$ irrespective of the value of $t_{s}$.

Exact discretization 
Let $A_{e}$ and $B_{e}$ represent the exact discretization of $A_{c}$ and $B_{c}$ respectively using (11). LMI (10) can then be rewritten as

$$
\left[\begin{array}{cc}
A_{e}^{T} P_{d} A_{e}-P_{d} & A_{e}^{T} P_{d} B_{e} \\
B_{e}^{T} P_{d} A_{e} & B_{e}^{T} P_{d} B_{e}
\end{array}\right] \leq\left[\begin{array}{cc}
Q & S \\
S^{T} & R
\end{array}\right] .
$$

However, from (11), the exact discretized cost retains the definiteness of the continuous-time cost. Hence, the right-hand-side of (16) is positive semi-definite and there exists at least one $P_{d}\left(P_{d}=0\right)$ that satisfies (16) and ensures dissipativity of the discrete-time setup.

Remark 3. Theorem 3.3 further shows the inherent robustness of the classical LQR (with a positive (semi)definite running) cost to discretization errors that could have resulted from sampling the system i.e., the dissipativity property is not lost through discretization. This does not however imply that the transient performance will not be affected by discretization errors.

A common notion in sampled-data theory is that if the sampling period, $t_{s}$, is sufficiently small such that terms in $t_{s}^{2}$ and higher in (11) and (12) can be neglected, then a first order approximation of the exact $\mathrm{ZOH}$ discretization approximates the exact $\mathrm{ZOH}$ discretization quite well and can be used in place of the full discretization [20-22]. However, while this holds in the case of a positive semi-definite cost, there is no guarantee that it holds when the cost function is indefinite as in the case of economic MPC.

\subsection{Example}

We conclude this section by using an example to illustrate the results obtained in this section. The origin is the optimal static equilibrium and the closed-loop behaviour of the system is simulated using a variable step-size ordinary differential equation (ODE) solver, ode45, in MATLAB with the relative and absolute error tolerances of $10^{-3}$ and $10^{-6}$ respectively.

We consider the system (3) with

$$
A_{c}=\left[\begin{array}{cc}
0 & 1 \\
-2 & -5
\end{array}\right], \quad B_{c}=\left[\begin{array}{l}
0 \\
1
\end{array}\right]
$$


and running cost (4) with

$$
Q_{c}=\left[\begin{array}{ll}
0 & 0 \\
0 & 0
\end{array}\right], \quad R_{c}=0.1, \quad S_{c}=\left[\begin{array}{l}
1 \\
1
\end{array}\right]
$$

The continuous time system is strictly dissipative with respect to the given cost function. On running Algorithm 3.1, it was observed that for sampling periods satisfying $0.4385 \leq t_{s} \leq 1.262$ and $0<t_{s} \leq 0.08$ the dissipativity of the system is preserved using the first order discrete approximation. The behaviour of the closed-loop system using different sampling periods (with a constant horizon $T$ ) when the controller is computed using MPC approach is simulated and shown in Figure 1. As shown, the closed-loop system is stable for sampling periods for which dissipativity is preserved $\left(t_{s}=0.5\right.$ and $\left.t_{s}=0.05\right)$ and unstable for $t_{s}=0.1$ which is outside the set of sampling intervals for which dissipativity is preserved.
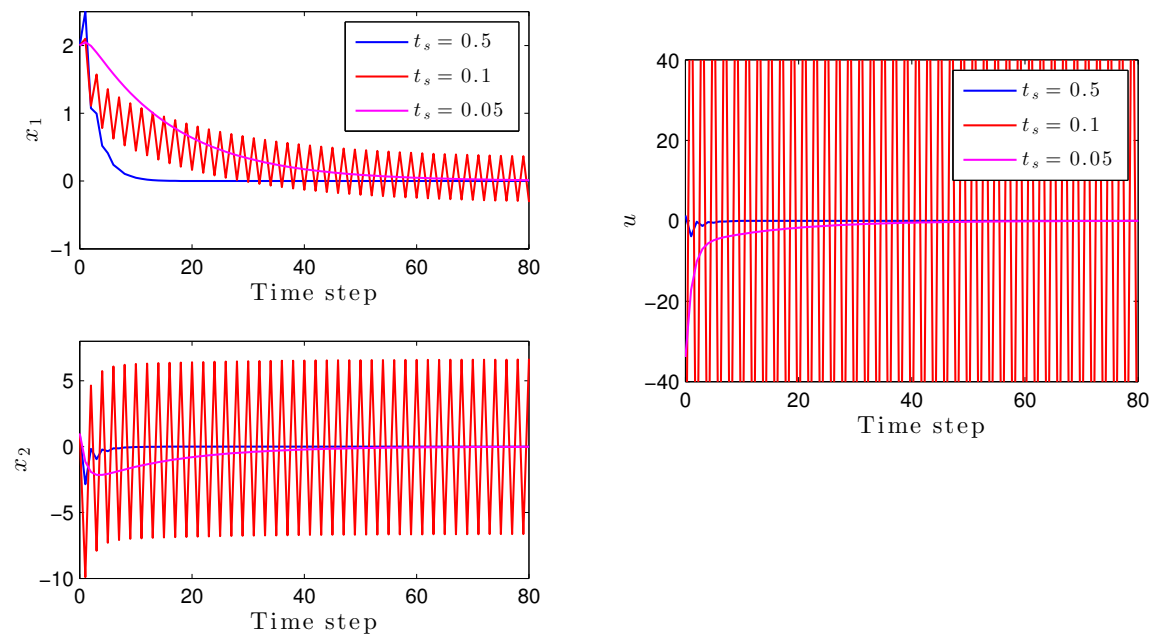

Figure 1: Comparison of the effect of different sampling periods using an initial condition $x(0)=[2,1]$ with a terminal equality constraint, $x(T)=x_{s}$. 


\section{Effect of Direct Transcription Method}

The general direct method approach to solving optimal control problems of form (1) is the use of direct transcription methods. The basic idea in direct transcription is to approximate the infinite-dimensional problem (1) as a finitedimensional nonlinear programming (NLP) problem of the form

$$
\min _{z}\{f(z) \mid b(z)=0, c(z) \geq 0\}
$$

where $z$ is a finite-dimensional vector representing the optimization degrees of freedom and $f, b$ and $c$, all differentiable functions [23]. The resulting NLP is then solved using NLP solvers. Variants of this method exist in literature [23-31] however, we will consider only the direct collocation approach in this paper. In this approach, the optimal control problem (OCP) is fully discretized by parameterizing the control and state profiles using piecewise polynomial functions over $M$ finite elements, $t_{0}<t_{1}<\ldots<t_{T}$ where the mesh size, $h_{i}=t_{i}-t_{i-1}$ and for equidistant meshes, $h=\frac{T}{M}$. The coefficients of these polynomial functions are then treated as optimization variables $(z)$, with the degree of the functions depending on the number of collocation points used, details of which can be found in $[23,32]$.

Of importance in direct collocation approach to solving (1) is whether the returned solution converges (and at what rate it converges) to the true solution of the optimal control problem (1). While we do not attempt to give any theoretical proof, we show that the returned solution from the resulting optimization problem, when using direct methods, may not converge to the true solution of the optimal control problem if the cost function is indefinite or nonconvex. This is in contrast to the convex cost case where convergence to the true solution can be shown, for different direct solution methods [33-38]. We also note however that no general convergence proof for direct collocation methods of solving constrained optimal control problems exists in the literature.

We consider the linear-quadratic Example 3.1 again. To implement the direct collocation method, we use the MATLAB-based Imperial College London 
Optimal Control Software (ICLOCS) [39] to transcribe the OCP and solve the resulting NLP using IPOPT [40], an interior point-based optimization algorithm. ICLOCS uses Lobatto IIIA methods (which are implicit Runge-Kutta methods) for collocation and the user has the option of selecting either trapezoidal (a second order Lobatto IIIA method) or Hermite-Simpson (a fourth order Lobatto IIIA method) discretization methods. The trapezoidal method parameterizes the state profiles using piecewise quadratic polynomials and the control profile with piecewise linear polynomials while the Hermite-Simpson method parameterizes the state profiles using cubic piecewise polynomials and control profile with quadratic piecewise polynomials.

The optimal control problem is defined as

$$
\begin{array}{ll}
\min _{\mathbf{u}} & \int_{0}^{25} l_{c}(x(t), u(t)) \mathrm{d} t \\
\text { s.t }\left\{\begin{array}{l}
\dot{x}=A_{c} x+B_{c} u . \\
x(0)=x_{0}, \quad x(25)=0 \\
-10 \leq x \leq 10, \quad-10 \leq u \leq 10 .
\end{array}\right.
\end{array}
$$

We note that the continuous time system is strictly dissipative with respect to the given cost function. The meshes have been chosen to be equidistant with the mesh size equal to the sampling interval, $t_{s}=0.5$.

The optimal control problem (18) is solved in a receding horizon manner and the first element of the optimal input sequence from (18) is applied to the continuous-time system at each time instant. The closed-loop behaviour of the system is then simulated using a variable step-size ODE solver, ode45, in Matlab. We compare the behaviour of the closed-loop system when (18) is solved using the trapezoidal (TR), Hermite-Simpson (HS) and first order approximation (described in section 3) discretization schemes. As shown in Figure 2, only the first order approximation scheme resulted in an asymptotically stable closed-loop system with TR yielding an oscillatory closed-loop behaviour and HS not converging to the origin.

With $t_{s} \leq 0.4$, the HS method resulted in an asymptotically stable closed- 

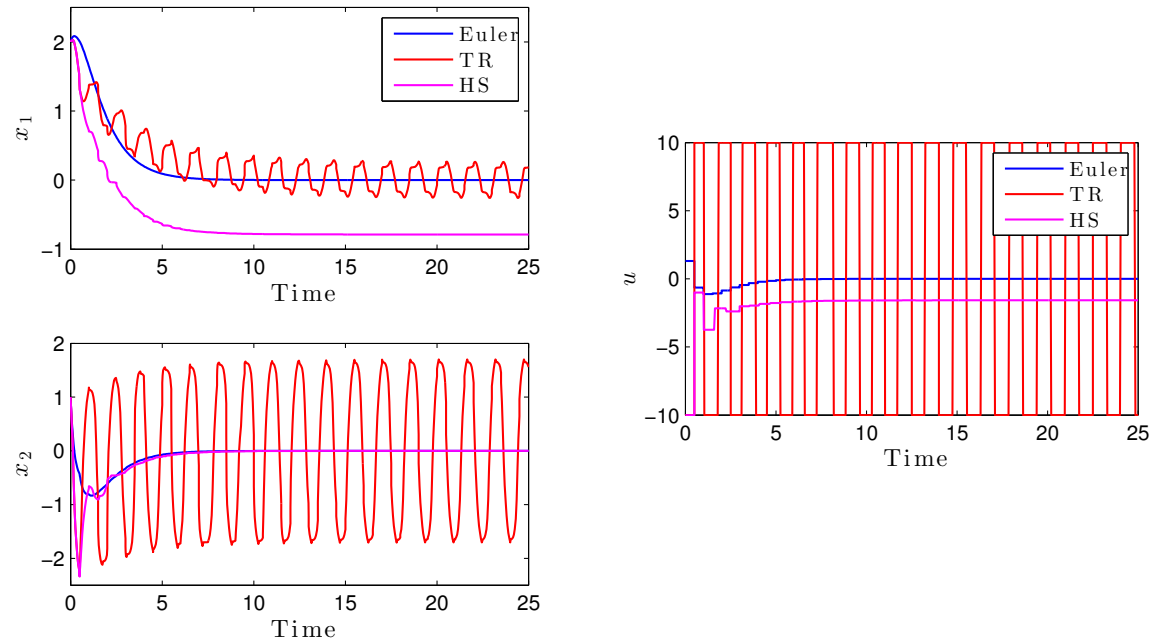

Figure 2: Comparison of the different discretization methods using an initial condition $x(0)=$ $[2,1] . t_{s}=0.5$.

loop system while for $t_{s}<0.2$ the TR method resulted in an asymptotically stable closed-loop system. However, with any of these sampling intervals, the first order approximation setup loses stability with Algorithm 3.1 showing that the discretized setup is only dissipative for sampling periods within the intervals $0<t_{s} \leq 0.08$ and $0.4385 \leq t_{s} \leq 1.262$. This is a linear system and yet different closed-loop performances result from different discrete approximations of (18) with a non-convex cost despite the fact that the continuous-time system is strictly dissipative with respect to the cost function. One could expect similar or even more complex behaviours with nonlinear systems. When unstable closedloop trajectories like those in Figure 2 are observed, it is usually assumed that the system is not dissipative with respect to the cost function especially when the system is nonlinear and it may not be easy to verify dissipativity of the continuous-time setup.

\subsection{Rotating the Running Cost}

The concept of 'rotated' stage cost is quite established in e-MPC literature for proving stability of the closed loop system in discrete-time setting. The 
rotated stage cost is defined as a function of the original cost and the storage function (assuming the system is strictly-dissipative). By showing that

1. The rotated cost is positive-definite provided the system is strictly dissipative with respect to the original cost function

2. Replacing the original cost with the rotated cost in the optimal control problem yields the same optimal input sequence,

the stability of the closed loop system is established $[4,5,17]$ by treating the optimal rotated cost function as a Lyapunov function. However, while this equivalence between the rotated and original cost functions (and hence stability) is proven for discrete-time systems, the examples given are usually continuoustime systems. In what follows, we show that though this equivalence also holds for the continuous-time case, there is no guarantee that it holds for the discrete approximation if the discretization is not properly done i.e., while the rotated cost function will result in an asymptotically stable closed-loop system, the original cost function can result in an unstable closed-loop system.

Let us assume a differentiable storage function $V(x)$ in Definition 1. Then system (3) is strictly dissipative with respect to running cost (4) if

$$
\frac{d V(x)}{d t}<l_{c}(x(t), u(t))-l_{c}\left(x_{s}, u_{s}\right)
$$

The rotated cost is defined as

$$
L_{c}(x(t), u(t))=l_{c}(x(t), u(t))-\frac{d V(x)}{d t}-l_{c}\left(x_{s}, u_{s}\right)
$$

which is positive-definite by definition. Replacing $l_{c}(\cdot, \cdot)$ with $L_{c}(\cdot, \cdot)$ in (1),

$$
\begin{aligned}
\tilde{J}(x, \mathbf{u}) & \triangleq \int_{0}^{T} L_{c}(x(t), u(t)) \mathrm{d} t \\
& =\int_{0}^{T}\left(l_{c}(x(t), u(t))-\frac{d V(x)}{d t}\right) \mathrm{d} t-l_{c}\left(x_{s}, u_{s}\right) \\
& =\int_{0}^{T}\left(l_{c}(x(t), u(t)) \mathrm{d} t-\left.\right|_{0} ^{T} V(x)-l_{c}\left(x_{s}, u_{s}\right)\right. \\
& =\int_{0}^{T}(l_{c}(x(t), u(t)) \mathrm{d} t+\underbrace{V(x(0))-V(x(T))}_{\text {independent of u }}-\underbrace{l_{c}\left(x_{s}, u_{s}\right)}_{\text {constant, from (2) }}
\end{aligned}
$$


Since the difference between $J$ in (1) and $\tilde{J}$ in (19) is independent of the optimization variable $(\mathbf{u})$ and both optimal control problems are subject to same constraints, it can be concluded that optimal control problems (1) and (19) have equivalent solution sets. Hence stability of the closed-loop system can be proven by showing that $\tilde{J}$ is a Lyapunov function for the closed-loop system.

We revisit the example in (18). A storage function $V(x)=x^{T} P_{c} x$ where $P_{c}=\left[\begin{array}{cc}6 & 1 \\ 1 & 0.5\end{array}\right]$ exists for which the continuous-time setup is dissipative. The time-derivative of $V(x)$ can be easily computed to get the rotated cost, $L_{c}(\cdot, \cdot)$, before discretization. Since the rotated cost is positive-definite, the trajectory obtained from the direct collocation is guaranteed to converge to the true solution of the optimal control problem. Closed-loop trajectories of the system using the second order Lobatto IIIA method are shown in Figure 3. As shown, while
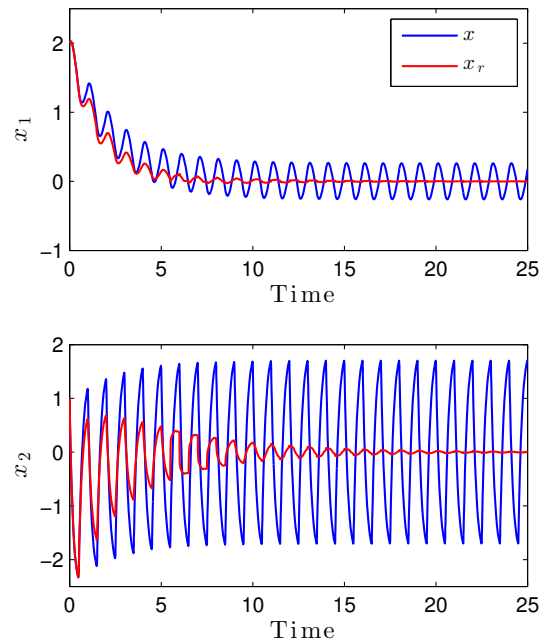

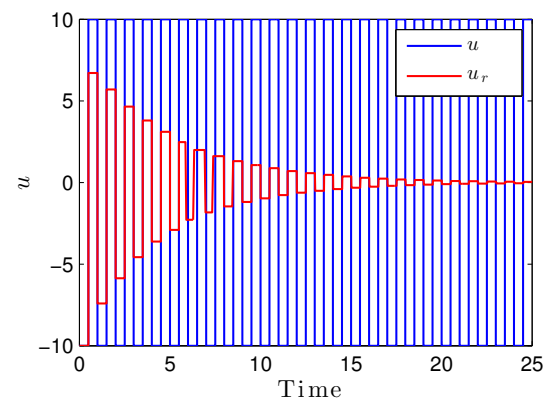

Figure 3: Closed loop trajectories based on trapezoidal discretization using original and rotated (subscript r) costs from initial condition $x(0)=[2,1]$.

the rotated cost yields a stable closed-loop system, the original cost resulted in an unstable closed-loop system, using the same mesh size of 0.5 .

While, for this particular example, a smaller mesh size could have resulted in a stable closed-loop trajectory for the trapezoidal discretization using the 
original cost function, we note that the use of $t_{s}=0.5$ is not the main problem as with this same mesh size, the rotated cost function yielded a stable closedloop system. Rather, the problem is the non-convexity of the cost function. If a storage function is known, perhaps the cost can be rotated before optimization is carried out. However, in the general case, the storage function is only assumed to exist. Moreover, though knowledge of the real value of a storage function is not essential for the asymptotic stability proof, it will be good practice as observed in this example to search for a storage function and rotate the stagecost before setting up the optimization problem, especially when approximating a continuous-time OCP.

\section{Economic MPC Example}

Having seen the possible pitfalls associated with solving the OCP (1) when the cost function is non-convex, we consider a popular example from e-MPC literature. The model considered is that of an isothermal continuous stirred tank reactor $[4,16]$ with the dynamical equations

$$
\begin{aligned}
\dot{c}_{a} & =\frac{u}{V_{R}}\left(c_{a f}-c_{a}\right)-k_{r} c_{a} \\
\dot{c}_{b} & =\frac{u}{V_{R}}\left(c_{b f}-c_{b}\right)+k_{r} c_{b}
\end{aligned}
$$

where $V_{R}=10 \mathrm{l}$ is the volume of the reactor, $c_{a f}=1 \mathrm{moll}^{-1}$ and $c_{b f}=0 \mathrm{moll}^{-1}$ are the feed concentrations with the rate constant, $k_{r}=0.41 \mathrm{~mol}^{-1} \mathrm{~min}^{-1}$. The input $u$ is the feed flow-rate. The states of the system are the molar concentrations of $c_{a}$ and $c_{b}$ and are constrained to $x_{1}, x_{2} \in[0,1]$ with the input constraint set $\mathbb{U}=\{u \in \mathbb{R}: 0 \leq u \leq 20\}$.

The economic objective of the process, based on price of product $x_{2}$ and a separation cost assumed directly proportional to the feed flow-rate [16], is given as

$$
l_{e}(x, u)=30-2 u x_{2}+0.5 u
$$

The optimal static equilibrium of the process with respect to this economic objective is $x_{s}^{*}=\left[\begin{array}{ll}0.5 & 0.5\end{array}\right]^{\prime}, u_{s}^{*}=4$. This example has been considered in 
different economic-MPC [4, 16, 41-43] and approximated economic-MPC [44] literature. In all these literature, the system was said to be non-dissipative with respect to the cost function and that the closed-loop system cannot be asymptotically stable unless the cost function is regularized. We note however that no rigorous dissipativity analysis of the system has been carried out.

\subsection{Approximated economic $M P C$}

Using the approximated e-MPC approach in [44], local information about the system is obtained from the second-order expansion of the system at its static equilibrium. The linear system is obtained as

$$
A_{c}=\left.\frac{\partial \dot{x}}{\partial x}\right|_{x_{s}} B_{c}=\left.\frac{\partial \dot{x}}{\partial u}\right|_{u_{s}}
$$

The Hessian of the running cost is also obtained as

$$
H=\left[\begin{array}{ll}
Q_{c} & S_{c} \\
S_{c}^{T} & R_{c}
\end{array}\right]=\left.\frac{\partial^{2} L\left(x, u, \lambda_{1}, \lambda_{2}\right)}{\partial z^{2}}\right|_{z_{s}}
$$

where $L(\cdot, \cdot, \cdot, \cdot)$ is the Lagrangian of the steady-state optimization problem,

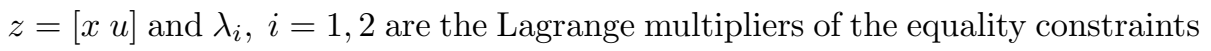
in the steady-state optimization problem. The local system can then be written in form of (3) as:

$$
\dot{\tilde{x}}=A_{c} \tilde{x}+B_{c} \tilde{u}
$$

where $\tilde{x}=x-x_{s}, \tilde{u}=u-u_{s}$,

$$
A_{c}=\left[\begin{array}{cc}
-0.8 & 0 \\
0.4 & -0.4
\end{array}\right], \quad B_{c}=\left[\begin{array}{c}
0.05 \\
-0.05
\end{array}\right]
$$

and running cost (4)

$$
l(\tilde{x}, \tilde{u})=\tilde{x}^{T} Q_{c} \tilde{x}+\tilde{u}^{T} R_{c} \tilde{u}+2 \tilde{x}^{T} S_{c} \tilde{u}
$$

with

$$
Q_{c}=\left[\begin{array}{ll}
0 & 0 \\
0 & 0
\end{array}\right], \quad R_{c}=0, \quad S_{c}=\left[\begin{array}{l}
1 \\
0
\end{array}\right]
$$


Though the Hessian is indefinite, a feasible $P_{c}=\left[\begin{array}{cc}26.84 & 6.84 \\ 6.84 & 6.84\end{array}\right]$ was found that satisfies LMI (6) which implies that the continuous-time local approximation is locally dissipative around the static equilibrium hence static equilibrium operation of the local system is optimal.

Using the first order discrete approximation discussed in Section 3 and running Algorithm 3.1, it was discovered that no sampling period is able to preserve the dissipativity of the system when using the first order approximation. However, using an exact discretization method $(\mathrm{ZOH})$ and $t_{s}=0.1$, the discretized setup was found to be locally dissipative.

Figure 4 shows the closed-loop trajectories of the local system from an initial state $x_{0}=\left[\begin{array}{ll}1 & 0.1\end{array}\right]$ when discretized using a sampling time $t_{s}=0.1$. As shown, the first order approximated setup is unstable as anticipated since no sampling period is able to preserve the dissipativity of the system. The exact-discretized system is in contrast asymptotically stable as shown.
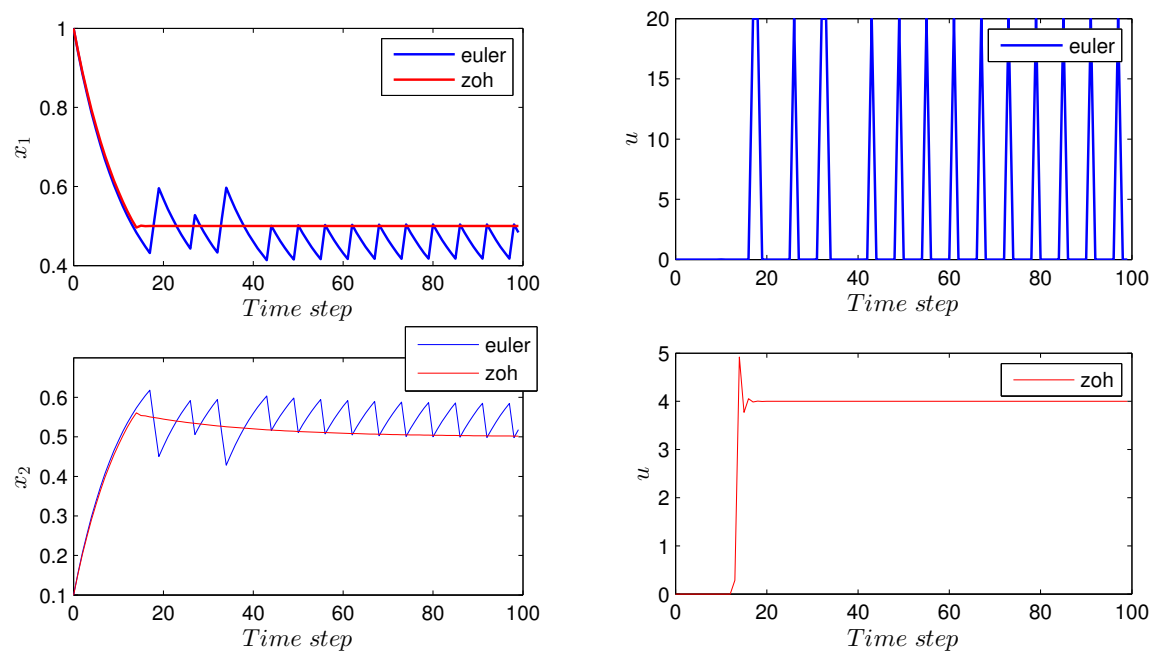

Figure 4: Comparison of closed-loop trajectories of the system using first order approximation and exact $\mathrm{ZOH}$ discretization. 


\subsection{Closed-loop Behaviour of the Full System}

Having considered the local behaviour of the system around the optimal static equilibrium and observed that the continuous-time system is locally dissipative with respect to the cost function around the static equilibrium, we now consider the real nonlinear system and objective function as defined in (20) and (21). The optimal control problem was set up with the system's dynamics described by (20) and the running cost by (21). The approximate discretization was obtained with direct collocation using a second order Lobatto IIIA method and the closed-loop behaviour of the system is simulated using a variable stepsize ODE solver, ode45, in Matlab. The time horizon, $T=20$ remains fixed in all simulations with the terminal constraint $x(T)=x_{s}$. The behaviour of the closed-loop system is considered in the case when the mesh size, $h$, is the same as the sampling interval, $t_{s}$, and when they are different. As shown in Figure 5 , with $t_{s}=0.5, h=0.1$, the closed loop system is asymptotically stable as against the unstable response obtained using $t_{s}=h=0.5$. Once again, no regularization of the cost function has been done here. The difference in both closed-loop trajectories is the way in which the discrete approximation was carried out. We note that the closed-loop system was not asymptotically stable for any value of $t_{s}$ chosen to be equal to $h$.

\section{Conclusion}

The effects of discretization of optimal control problems employing economic objective functions have been investigated in this paper. Starting with the explicit first order approximation of an exact discretization scheme, we showed the effect of the sampling period and discretization method on dissipativity (hence stability of the closed-loop system) when moving from a continuoustime setup to a discrete-time setup. The importance of incorporating a storage function (when available) was also highlighted. A popular example from the economic MPC literature was used to illustrate these results. 

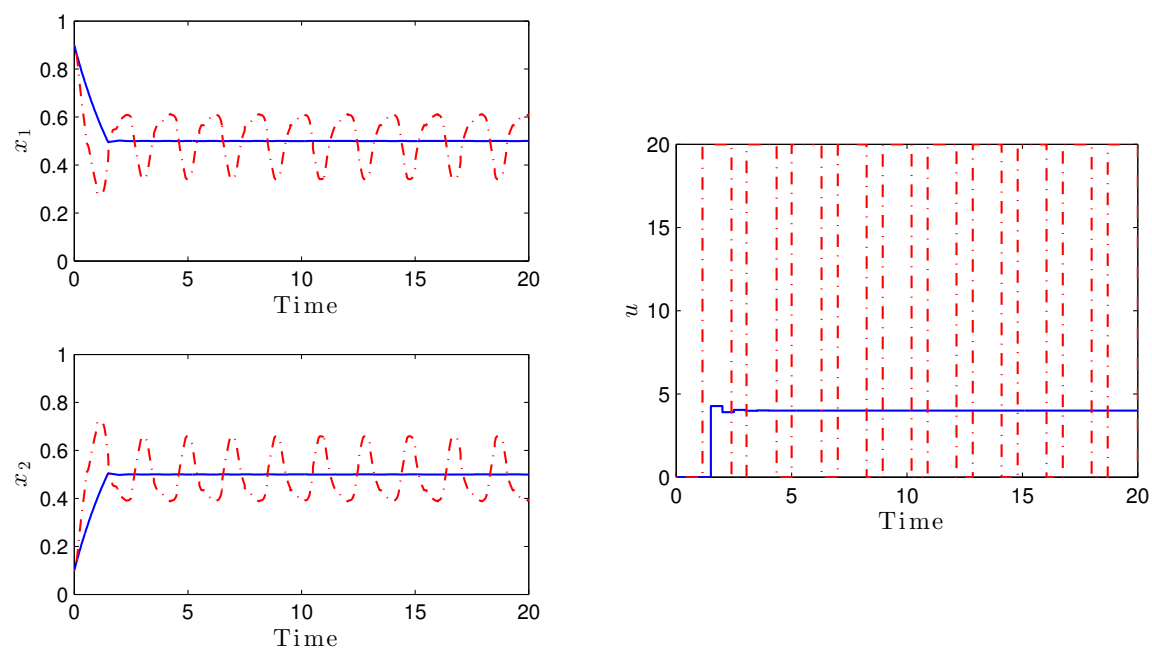

Figure 5: Closed-loop trajectories of the system with $t_{s}=h=0.5$ (dashed) and $t_{s}=0.5, h=$ 0.1 (solid) from an initial condition $x(0)=[2,1]$.

\section{Acknowledgements}

This research is funded by the Federal Government of Nigeria through the Presidential Special Scholarship Scheme for Innovation and Development.

\section{References}

[1] T. Chen, B. A. Francis, Optimal sampled-data control systems, Springer, 1995.

[2] K. J. Aström, B. Wittenmark, Computer-controlled Systems (3rd Ed.), Prentice-Hall, Inc., Upper Saddle River, NJ, USA, 1997.

[3] G. F. Franklin, M. L. Workman, D. Powell, Digital Control of Dynamic Systems, 3rd Edition, Addison-Wesley Longman Publishing Co., Inc., Boston, MA, USA, 1997.

[4] R. Amrit, J. B. Rawlings, D. Angeli, Economic optimization using model predictive control with a terminal cost, Annual Reviews in Control 35 (2) (2011) $178-186$. 
[5] D. Angeli, R. Amrit, J. Rawlings, On average performance and stability of economic model predictive control, IEEE Transactions on Automatic Control 57 (7) (2012) 1615-1626.

[6] M. A. Muller, D. Angeli, F. Allgower, On convergence of averagely constrained economic MPC and necessity of dissipativity for optimal steadystate operation, in: American Control Conference (ACC), 2013, 2013, pp. 3141-3146.

[7] T. Faulwasser, M. Korda, C. Jones, D. Bonvin, Turnpike and dissipativity properties in dynamic real-time optimization and economic MPC, in: Proceedings of the 53rd IEEE Conference on Decision and Control, 2014, pp. $2734-2739$.

[8] R. Costa-Castelló, E. Fossas, On preserving passivity in sampled-data linear systems, European Journal of Control 13 (6) (2007) 583 - 590.

[9] J. Jiang, Preservations of positive realness under discretizations, Journal of the Franklin Institute 330 (4) (1993) 721 - 734.

[10] Y. Oishi, Passivity degradation under the discretization with the zero-order hold and the ideal sampler, in: Proceedings of the 49th IEEE Conference on Decision and Control, 2010, pp. 7613-7617.

[11] D. S. Laila, D. Nešić, A. R. Teel, Open- and closed-loop dissipation inequalities under sampling and controller emulation, European Journal of Control 8 (2) (2002) $109-125$.

[12] J. C. Willems, Dissipative dynamical systems, European Journal of Control 13 (2007) $134-151$.

[13] N. Kottenstette, P. J. Antsaklis, Relationships between positive real, passive dissipative \& positive systems, in: American Control Conference (ACC), 2010, IEEE, 2010, pp. 409-416. 
[14] G. C. Goodwin, K. S. Sin, Adaptive Filtering Prediction and Control, Englewood Cliffs, New Jersey 07632: Prentice-Hall, Inc., 1984.

[15] C. Scherer, S. Weiland, Linear matrix inequalities in control, Lecture Notes, Dutch Institute for Systems and Control, Delft, The Netherlands.

[16] M. Diehl, R. Amrit, J. Rawlings, A Lyapunov Function for Economic Optimizing Model Predictive Control, IEEE Transactions on Automatic Control 56 (3) (2011) 703-707.

[17] J. Rawlings, D. Angeli, C. Bates, Fundamentals of economic model predictive control, in: Proceedings of the 51st IEEE Conference on Decision and Control, 2012, pp. 3851-3861.

[18] G. Pannocchia, J. B. Rawlings, D. Q. Mayne, W. Marquardt, On computing solutions to the continuous time constrained linear quadratic regulator, Automatic Control, IEEE Transactions on 55 (9) (2010) 2192-2198.

[19] G. Pannocchia, J. B. Rawlings, D. Q. Mayne, G. M. Mancuso, Whither discrete time model predictive control?, Automatic Control, IEEE Transactions on 60 (1) (2015) 246-252.

[20] A. H. Levis, R. A. Schlueter, M. Athans, On the behaviour of optimal linear sampled-data regulators, International Journal of Control 13 (2) (1971) 343-361.

[21] S. Melzer, B. Kuo, Sampling period sensitivity of the optimal sampled data linear regulator, Automatica 7 (3) (1971) 367 - 370.

[22] S. L. Osburn, D. S. Bernstein, An exact treatment of the achievable closedloop $\mathrm{H}_{2}$ performance of sampled-data controllers: from continuous-time to open-loop, Automatica 31 (4) (1995) 617 - 620.

[23] M. Diehl, H. Bock, H. Diedam, P.-B. Wieber, Fast Direct Multiple Shooting Algorithms for Optimal Robot Control, in: Fast Motions in Biomechanics and Robotics, Heidelberg, Germany, 2005. 
[24] P. I. Barton, R. J. Allgor, W. F. Feehery, S. Galán, Dynamic optimization in a discontinuous world, Industrial \& Engineering Chemistry Research 37 (3) (1998) 966-981.

[25] V. S. Vassiliadis, R. W. H. Sargent, C. C. Pantelides, Solution of a class of multistage dynamic optimization problems. 1. problems without path constraints, Industrial \& Engineering Chemistry Research 33 (9) (1994) $2111-2122$.

[26] H. Bock, K. Plitt:, A multiple shooting algorithm for direct solution of optimal control problems, in: 9th IFAC World Congress, Budapest, Pergamon Press, 1984, pp. 243-247.

[27] D. Leineweber, Efficient Reduced SQP Methods for the Optimization of Chemical Processes Described by Large Sparse DAE Models, FortschrittBerichte VDI.: Verfahrenstechnik, VDI-Verlag, 1999.

[28] J. Betts, P. Frank, A sparse nonlinear optimization algorithm, Journal of Optimization Theory and Applications 82 (3) (1994) 519-541.

[29] J. T. Betts, W. P. Huffman, Application of sparse nonlinear programming to trajectory optimization, Journal of Guidance, Control, and Dynamics 15 (1) (1992) 198-206.

[30] A. Cervantes, L. T. Biegler, Large-scale DAE optimization using a simultaneous NLP formulation, AIChE Journal 44 (5) (1998) 1038-1050.

[31] A. M. Cervantes, A. Wächter, R. H. Tütüncü, L. T. Biegler, A reduced space interior point strategy for optimization of differential algebraic systems, Computers \& Chemical Engineering 24 (1) (2000) 39 -51.

[32] J. Betts, Practical Methods for Optimal Control and Estimation Using Nonlinear Programming, 2nd Edition, Society for Industrial and Applied Mathematics, 2010. 
[33] W. W. Hager, Rates of convergence for discrete approximations to unconstrained control problems, SIAM Journal on Numerical Analysis 13 (4) (1976) 449-472.

[34] W. W. Hager, Runge-kutta methods in optimal control and the transformed adjoint system, Numerische Mathematik 87 (1999) 247-282.

[35] S. Kameswaran, L. T. Biegler, Convergence rates for direct transcription of optimal control problems using collocation at radau points, Computational Optimization and Applications 41 (1) (2008) 81-126.

[36] K. Malanowski, C. Büskens, H. Maurer, Convergence of approximations to nonlinear optimal control problems, Lecture Notes in Pure and Applied Mathematics (1997) 253-284.

[37] A. L. Dontchev, W. W. Hager, The Euler approximation in state constrained optimal control, Math. Comput. 70 (233) (2001) 173-203.

[38] L. Han, M. K. Camlibel, J.-S. Pang, W. M. H. Heemels, A unified numerical scheme for linear-quadratic optimal control problems with joint control and state constraints, Optimization Methods and Software 27 (4-5) (2012) 761799.

[39] E. V. Wyk, P. Falugi, E. C. Kerrigan, Imperial college london optimal control software (ICLOCS) (2010).

[40] A. Wächter, L. T. Biegler, On the implementation of an interior-point filter line-search algorithm for large-scale nonlinear programming, Mathematical Programming 106 (1) (2006) 25-57.

[41] L. Fagiano, A. R. Teel, On generalized terminal state constraints for model predictive control, CoRR abs/1207.0788.

[42] L. Grune, M. Stieler, A Lyapunov function for economic MPC without terminal conditions, in: Proceedings of the 53rd IEEE Conference on Decision and Control, 2014, pp. 2740-2745. 
[43] A. Alessandretti, A. P. Aguiar, C. Jones, An economic model predictive control scheme with terminal penalty for continuous-time systems, in: Proceedings of the 53rd IEEE Conference on Decision and Control, 2014, pp. $2728-2733$.

[44] M. Zanon, S. Gros, M. Diehl, Indefinite linear MPC and approximated economic MPC for nonlinear systems, Journal of Process Control 24 (8) (2014) $1273-1281$. 